\title{
Respiratory Symptoms in Preterm Infants: Burden of Disease in THE FIRST YEAR OF LIFE
}

\author{
I. A. Pramana ${ }^{1 a}$, P. Latzin ${ }^{1 a, 2}$, L. J. Schlapbach ${ }^{1 b}$, G. Hafen ${ }^{1 a, 3}$, C. E. Kuehni², M. Nelle ${ }^{1 b}$, T. Riedelec, \\ U. Frey ${ }^{1 \mathrm{a}}$ \\ ${ }^{1}$ Dept. of Paediatrics, University of Bern, Switzerland, \\ aDivision of Paediatric Pulmonology, bDivision of Neonatology, ${ }^{\mathrm{C} D i v i s i o n}$ of Paediatric Intensive Care; \\ ${ }^{2}$ Institute of Social and Preventive Medicine (ISPM), University of Bern, Switzerland; \\ ${ }^{3}$ Dept. of Paediatrics, Division of Paediatric Pulmonology, University of Lausanne, Switzerland
}

\begin{abstract}
Objective: While respiratory symptoms in the first year of life are relatively well described for term infants, data for preterm infants are scarce. We aimed to describe the burden of respiratory disease in a group of preterm infants with and without bronchopulmonary dysplasia (BPD) and to assess the association of respiratory symptoms with perinatal, genetic and environmental risk factors.

Methods: Single centre birth cohort study: prospective recording of perinatal risk factors and retrospective assessment of respiratory symptoms during the first year of life by standardised questionnaires. Main outcome measures: Cough and wheeze (common symptoms), re-hospitalisation and need for inhalation therapy (severe outcomes). Patients: 126 preterms (median gestational age 28.7 weeks; 78 with, 48 without BPD) hospitalised at the University Children's Hospital of Bern, Switzerland 1999-2006.

Results: Cough occurred in $80 \%$, wheeze in $44 \%$, rehospitalisation in $25 \%$ and long term inhalation therapy in wheezers in 13\% of the preterm infants. Using logistic regression, the main risk factor for common symptoms was frequent contact with other children. Severe outcomes were associated with maximal peak inspiratory pressure, arterial cord blood $\mathrm{pH}$, APGARand CRIB-Score.

Conclusions: Cough in preterm infants is as common as in term infants, whereas wheeze, inhalation therapy and re-hospitalisations occur more often. Severe outcomes are associated with perinatal risk factors. Preterm infants who did not qualify for BPD according to latest guidelines also showed a significant burden of respiratory disease in the first year of life.
\end{abstract}

Key words: premature birth, cough, wheeze, risk factors, bronchopulmonary dysplasia.

\section{INTRODUCTION}

Respiratory problems are major consequences of prematurity, however the heterogeneity of respiratory sequelae and varying definitions have made a systematic assessment difficult. Northway et al. were the first to describe them as "bronchopulmonary dysplasia"
(BPD) [1]. The definition of BPD has been revised several times as with advances in perinatal treatment the clinical and pathomorphological patterns have changed. The most recent consensus definition of 2001 groups severity of "new BPD" into mild, moderate and severe according to gestational age and duration of supplementary oxygen [2]. Recently a large American cohort study used this definition to assess its usefulness for predicting respiratory morbidity in the first year of life [3]. The authors found a high sensitivity using this BPD classification, but specificity was poor: $30 \%$ of preterm infants had severe respiratory problems without qualifying for BPD. Since information on additional risk factors is lacking, it remains unclear whether the identification of risk factors could help improving prediction of respiratory morbidity after preterm birth.

Incidence and severity of respiratory symptoms in the first year of life are relatively well described in term born infants. One study reported persistent cough to occur in $56 \%$ and wheeze in $45 \%$ of termborn infants in the USA [4]. Another study, conducted in Australia, found $77 \%$ of the infants coughing and $27 \%$ wheezing during the first year of life [5]. In a recent prospective study from our group assessing respiratory morbidity in a birth cohort of term-born infants, any cough occurred in $93 \%$ and wheeze in $21 \%$ of the infants during the first year of life [6]. Frequency of symptoms was associated with male sex, higher birthweight, pre- and postnatal smoke exposure and day care or older siblings. Only 3 of all 195 infants $(1.5 \%)$ had to be hospitalised for respiratory problems in their first year of life [6].

In contrast to term infants, few data are available on respiratory morbidity in preterm infants. There are studies on outcome and associated risk factors from the pre-surfactant era [7], but data including children with the "new" form of BPD are scarce. Most of these studies focused on severe outcomes [3] and only few have determined risk factors [8] for pulmonary sequelae for which preterm infants show a predisposition [9-11].

The aim of the present study was to describe frequency and spectrum of respiratory symptoms during the first year of life in a contemporary population of 
preterm infants with and without BPD and to identify environmental and perinatal risk factors associated with common and severe respiratory symptoms.

\section{METHODS}

\section{STUdy DESIGN}

Preterm infants (gestational age $<37$ wks), born between July 1999 and June 2005 and hospitalised at the University Hospital of Bern, Switzerland, were prospectively recruited during a consultation of a paediatric pulmonologist before discharge. Perinatal data were routinely recorded using the institutional neonatology database (NEODAT 4.10, Tübingen, Germany). After the first year of life, a standardised questionnaire was sent to the parents to assess respiratory morbidity during the first year after discharge and ongoing environmental exposures.

\section{SubJECTS}

We approached preterm infants independent of their birthweight with 168 being recruited for the study. More parents of infants with lower birthweight agreed to participate resulting in a study population of 123 $(72 \%), 67(40 \%)$ and $3(2 \%)$ preterms with birthweights below 1500g, below $1000 \mathrm{~g}$ and below 500g, respectively.

Based on the ATS definition of BPD, we categorised preterm infants into healthy, mild, moderate and severe BPD, respectively [2]. BPD was defined as supplemental oxygen requirement for at least 28 days. Time point of assessment for BPD severity was at 36 weeks post-menstrual age (PMA) or at discharge (whichever occurred first) in infants born at less than 32 weeks of gestation, and at day 56 of life in infants born with at least 32 weeks of gestation. Mild BPD was defined as breathing room air, moderate BPD as requiring less than $30 \%$ supplemental oxygen and severe BPD as the need for more than $30 \%$ of oxygen. The standardised internal hospital guidelines were applied to determine the exact amount of oxygen needed (aiming for $92 \%$ or more oxygen saturation allowing dips to $86 \%$ ) and BPD classification was verified in each case by review of the medical records. The ethics committee of the canton of Bern approved the study protocol and written informed consent was obtained from the parents.

\section{Assessment of Perinatal Data}

The data routinely entered in the institutional database (NEODAT) are given in Table 1 and 3.

\section{DEFINITION OF RESPIRATORY MORBIDITY (OuTCOMES)}

Respiratory morbidity was assessed by a standardised questionnaire using modified questions from the International Study on Asthma and Allergies in Childhood (ISAAC) $[12,13]$ to the parents. The main outcomes were categorised into common and severe: Cough with cold ("did your child cough during times with a cold?") and wheeze were considered as com- mon respiratory symptoms, whereas re-hospitalisation for pulmonary reasons (excluding readmissions for diagnostic purposes) or long-term inhalation therapy (longer than four weeks) in wheezing infants were defined as severe outcomes $[3,14]$. In addition the subjective burden of disease and the need for consultations at any healthcare institution were assessed by the questionnaire.

\section{DEFINITION OF RISK FACTORS}

Potential risk factors were grouped into 3 categories:

A) Perinatal risk factors: Factors that have previously been described as either influencing the outcome of preterm infants or having an effect on the occurrence of BPD such as gestational age, sex, birthweight, maternal chorioamnionitis (histopathologically confirmed), PDA (patent ductus arteriosus) when of hemodynamic relevance in echocardiography, peak inspiratory pressure (PIP) during mechanical ventilation and antenatal steroids [15-18].

B) Factors of postnatal adaptation: Perinatal parameters reflecting the adaptation and initial condition of the newborn, such as APGAR score, arterial cordblood pH-value [19] and CRIB score.

C) Environmental and sociodemographic exposures: Known risk factors for respiratory symptoms in term infants including day care or older siblings, history of parental atopic disease and smoke exposure [6, 20].

\section{StATISTICAL ANALYSIS}

We used descriptive statistics to illustrate the occurrence and parental perception of respiratory morbidity. Second, to identify possible risk factors, we grouped all outcome parameters into binary categories and calculated the association between outcomes and risk factors using logistic regression analysis. All listed risk factors were entered into the model one by one as explanatory factors; continuous variables were centred. Results are given as odds ratios with confidence intervals and exact p-values. Due to the small sample size and a strong co-linearity between many of the parameters, only univariable analysis was performed. Sensitivity analysis stratifying for gestational age, sex, birthweight and age at reply of questionnaire confirmed the main results. Data analyses were performed using STATA ${ }^{\circledR}$ version 8.2 for Windows (STATA Corporation, College Station, TX, USA).

\section{RESULTS}

SUBJECTS

We primarily recruited 168 infants and received 126 $(75 \%)$ completed questionnaires back. Median gestational age was 28.7 weeks (range 23.9-36.1), median birthweight was 1070g (range 420-2980), 71 (56\%) infants were male and $55(44 \%)$ female; 78 (62\%) fulfilled the criteria of new BPD and 48 (38\%) were classified as having no BPD.

Of all questionnaires, $65 \%$ were filled in by the parents when their child was $1-3$ years, $35 \%$ when the child was older. A sensitivity analysis showed no dif- 
Table 1. Characteristics of the study population of preterm infants $(n=126)$.

\begin{tabular}{lcc}
\hline Characteristics & $\mathbf{n}$ & $\mathbf{\%}$ a \\
\hline Bronchopulmonary dysplasia (BPD) & 78 & 62 \\
$\quad$ mild BPD & 28 & 36 \\
$\quad$ moderate BPD & 36 & 46 \\
$\quad$ severe BPD & 28.71 & 18 \\
Gestational age in weeks (median/range) & 71 & $23.9-36.1$ \\
Male sex & 1070 & $420-2980$ \\
Birthweight in g (median/range) & 42 & 37 \\
Chorioamnionitis & 29 & 23 \\
Patent ductus arteriosus & 102 & 81 \\
Prenatal steroids & 48 & 38 \\
Surfactant therapy & 66 & 52 \\
Mechanical ventilation & 1 & $0-63$ \\
Days of mech. ventilation (median/range) & 113 & 90 \\
CPAP b & 42 & $0-385$ \\
Days on oxygen therapy (median/range) & 70 & $7-141$ \\
Days of initial hospital stay (median/range) & 75 & 61 \\
Contact to other children c & 13 & 10 \\
Maternal smoking in pregnancy d & 20 & 16 \\
Maternal atopy e &
\end{tabular}

a Data are given as number (percentage) if not indicated otherwise.

$\mathrm{b} \mathrm{CPAP}=$ continuous positive airway pressure breathing support

c Contact to other children $=$ having siblings or going to a nursery care institution regularly

$\mathrm{d}$ Assessed by parental questionnaire

e Maternal atopy $=$ mother has asthma and/or allergic rhinitis and/or atopic dermatitis

Table 2. Respiratory outcome in the first year after discharge.

\begin{tabular}{|c|c|c|c|c|c|c|c|c|c|c|}
\hline \multirow{3}{*}{ Variables } & \multicolumn{3}{|c|}{ total } & \multicolumn{3}{|c|}{ no BPD } & \multicolumn{3}{|c|}{ BPD } & \multirow{3}{*}{$\begin{array}{l}\text { BPD vs } \\
\text { no BPD }\end{array}$} \\
\hline & \multicolumn{3}{|c|}{$\mathrm{n}=126$} & \multicolumn{3}{|c|}{$\mathrm{n}=48$} & \multicolumn{3}{|c|}{$\mathrm{n}=78$} & \\
\hline & abs & $\%$ & $\mathrm{CI} \%$ & abs & $\%$ & $\mathrm{CI} \%$ & abs & $\%$ & $\mathrm{CI} \%$ & \\
\hline $\begin{array}{l}\text { Common outcomes } \\
\text { Cough }\end{array}$ & 100 & 79 & $71-86$ & 40 & 83 & $70-93$ & 60 & 77 & $66-86$ & 0.50 \\
\hline Wheezing $^{\mathrm{a}}$ & 56 & 44 & $36-54$ & 23 & 48 & $33-63$ & 33 & 43 & $31-54$ & 0.58 \\
\hline $\begin{array}{l}\text { Severe outcomes } \\
\text { Re-hospitalisation } \mathrm{b}\end{array}$ & 31 & 25 & $17-33$ & 11 & 23 & $12-37$ & 20 & 26 & $16-37$ & 0.83 \\
\hline Inhalation therapyc & 16 & 13 & $7-20$ & 3 & 6 & $1-17$ & 13 & 17 & $9-27$ & 0.25 \\
\hline
\end{tabular}

Numbers are given as absolute count (abs) as well as percentage and 95\% Confidence interval (CI).

a Data not available for 2 infants

${ }^{b}$ Rehospitalisation for respiratory reasons (excluding admissions for diagnostic purposes)

${ }^{\mathrm{c}}$ Inhalation therapy because of prolonged wheezing with corticosteroids, beta-mimetika or both, for more than four weeks.

ferences between those two groups. Descriptive data of the study group are given in Table 1.

\section{Burden of Respiratory Morbidity}

The occurrence of common respiratory symptoms (e.g. cough and wheeze) and severe outcomes (e.g. rehospitalisation and long term inhalation therapy) is shown in Table 2, burden of disease is illustrated in
Figure 1. Common respiratory symptoms occurred with high frequency: $80 \%(95 \%$-CI $71-86 \%)$ of the infants had cough and 44\% (95\%-CI 36-54\%) wheeze.

Severe outcomes were less frequent: re-hospitalisation occurred in $25 \%(95 \%$-CI $17-33 \%)$ of all children, and long term inhalation because of wheezing in $13 \%(95 \%$-CI $7-20 \%)$ of the infants. Parental statement of re-hospitalisation was very consistent with medical records. 


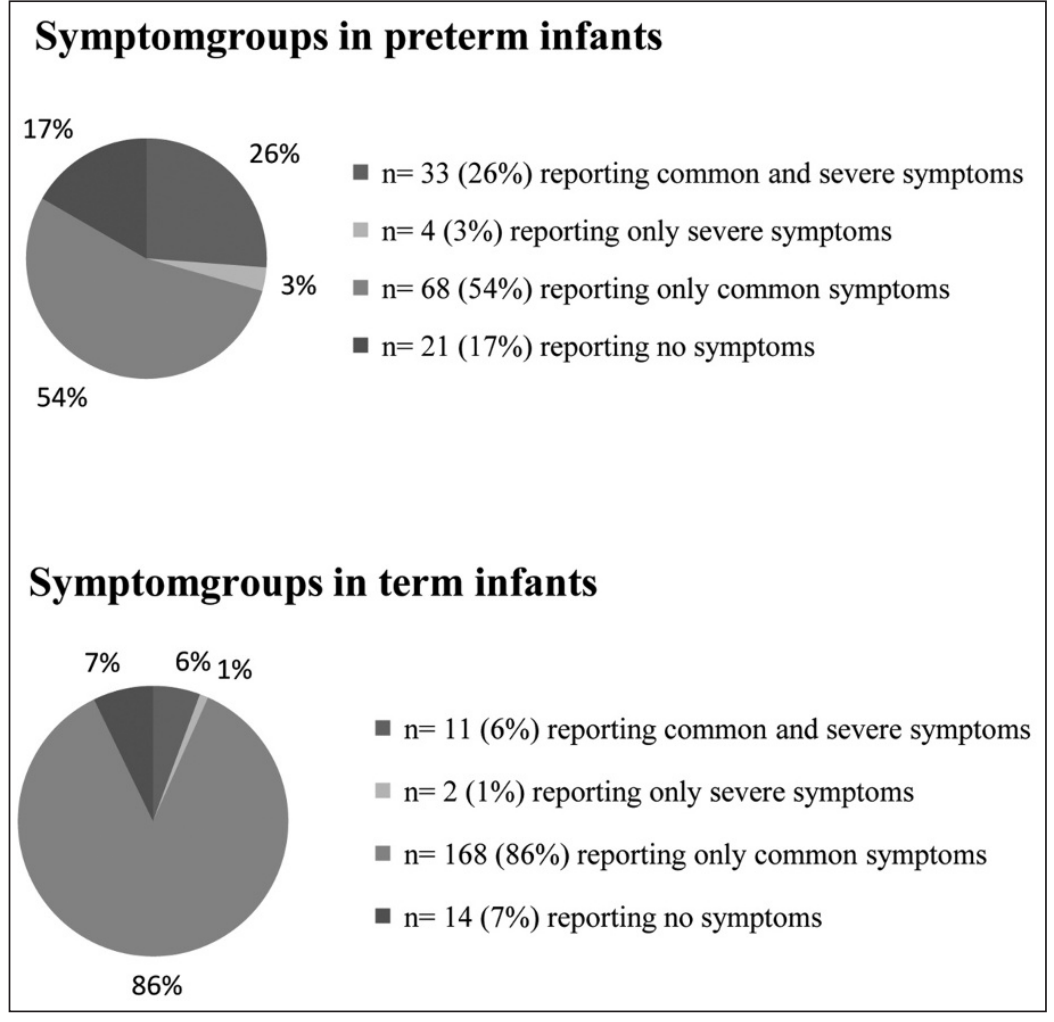

Fig. 1. Burden of common and severe symptoms in pretem $(\mathrm{n}=126)$ and term $(n=195)$.
While frequency of common symptoms did not differ between children with and without BPD, there was a trend towards more inhalation therapy in the BPD group.

Of the patients included $9(7 \%)$ had RSV vaccination; $8(6 \%)$ children suffered from severe RSV infection they needed to be hospitalised for, two of those had been vaccinated before.

\section{Parental Perception of Respiratory Morbidity}

In 53 families (45\%) parents considered their infant to cough as much as a healthy term infant, $34(29 \%)$ believed their infant to cough more often and 30 (26\%) less often than healthy infants.

Slightly more than half of the parents $(n=65,55 \%)$ reported that they consulted the community paediatrician in an emergency situation during the first year of life, $43(73 \%)$ of those once or twice and $16(27 \%)$ more often, up to a maximum of 10 times.

\section{DETERMINANTS OF RESPIRATORY OUTCOME}

Table 3 shows associations between analysed risk factors and respiratory morbidity.

\section{Common symptoms}

Common respiratory symptoms were mainly associated with environmental exposures. Children who were exposed to other children had a four-fold risk of having common symptoms (OR 4.7; 95\%-CI 1.8-12.6, $\mathrm{p}=0.002)$. While there was no difference between the outcomes cough and wheeze for most of the risk factors, maternal smoking during pregnancy was associated mainly with wheeze (OR 4.7; 95\%-CI 1.2-18.1, p =
$0.024)$ and contact with other children mainly with cough in the first year of life (OR 4.1; 95\%-CI 1.610.5, $\mathrm{p}=0.004)$.

\section{Severe outcomes}

Contrary to those for common symptoms, risk factors for severe outcomes were mainly parameters representing perinatal adaptation. A significant association with both re-hospitalisation and inhalation therapy was found for high peak inspiratory pressure, low arterial cord blood $\mathrm{pH}$, low APGAR-score and low CRIBscore, as shown in Table 3. Low arterial cord blood $\mathrm{pH}(<7.15)$ was associated with a 4.4 -fold $(95 \%$-CI 1.4-13.6, $\mathrm{p}=0.011)$ increased likelihood of severe symptoms.

No association was found between any of the investigated outcomes and need for intubation, surfactant therapy, parental educational status or parental smoking in the first year.

\section{Discussion}

\section{SUMMARY}

In our cohort of preterm infants with and without $\mathrm{BPD}$, respiratory symptoms were frequent during infancy: cough with cold was reported for $80 \%$ and wheeze for $45 \%$ of infants, respectively. More severe symptoms were less common, with re-hospitalisation for respiratory reasons occurring in $25 \%$ of infants and $13 \%$ of infants needing long-term inhalation therapy of more than four weeks because of wheezing.

Risk factors differed between common and severe symptoms. While common symptoms were associated with environmental factors such as contact to other children, severe symptoms were associated with risk 
Table 3. Risk factors and their association with common and severe respiratory outcomes in all subjects ( $\mathrm{n}=126)$.

\begin{tabular}{|c|c|c|}
\hline \multirow[t]{2}{*}{ Risk factors } & $\begin{array}{l}\text { Common outcomes } \\
\qquad \mathrm{n}=101\end{array}$ & $\begin{array}{c}\text { Severe outcomes } \\
\mathrm{n}=37\end{array}$ \\
\hline & OR CI p-value & CI p-value \\
\hline $\mathrm{BPD}^{\mathrm{a}}$ & $0.57(0.22-1.49) \quad 0.249$ & $1.20(0.54-2.66) 0.659$ \\
\hline \multicolumn{3}{|l|}{ Known risk factors for $B P D$} \\
\hline Gestational age, per week & $1.04(0.89-1.22) 0.605$ & $0.94(0.82-1.08) 0.375$ \\
\hline Male sex & $1.25(0.52-3.00) 0.625$ & $1.02(0.47-2.22) 0.953$ \\
\hline Birthweight, per kg & $1.36(0.56-3.29) 0.493$ & $0.70(0.32-1.51) 0.362$ \\
\hline Chorioamnionitis $^{b}$ & $1.58(0.56-4.44) 0.387$ & $1.55(0.68-3.52) 0.295$ \\
\hline $\mathrm{PDA}^{\mathrm{C}}$ & $1.65(0.51-5.32) 0.402$ & $0.56(0.21-1.52) 0.253$ \\
\hline PIPd & $1.10(0.92-1.33) 0.292$ & $1.24(1.05-1.47) 0.012$ \\
\hline \multicolumn{3}{|l|}{ Factors of perinatal adaption } \\
\hline Cord blood $\mathrm{pH}^{\mathrm{e}}$ & $1.98(0.41-9.48) 0.393$ & $4.37(1.4-13.61) 0.011$ \\
\hline APGAR score at 10 min. ${ }^{f}$ & $1.05(0.74-1.49) 0.783$ & $1.55(1.14-2.12) 0.006$ \\
\hline CRIB-score & $0.93(0.82-1.05) 0.232$ & $1.14(1.01-1.28) 0.024$ \\
\hline \multicolumn{3}{|l|}{ Environmental risk factors } \\
\hline Contact to other childreng & $4.71(1.77-12.56) 0.002$ & $1.30(0.59-2.90) 0.516$ \\
\hline Maternal atopy & $1.52(0.41-5.66) 0.532$ & $0.82(0.27-2.46) \quad 0.727$ \\
\hline Smoking in pregnancy & $1.41(0.29-6.79) \quad 0.672$ & $1.58(0.48-5.20) 0.450$ \\
\hline
\end{tabular}

In the table are shown: Odds ratio (OR), calculated by univariable logistic regression, defining the increased odds of having the respective outcome with the respective risk factor present; $95 \%$ Confidence interval (CI) and p-value.

a BPD was defined as in the consensus guidelines (2)

b Chorioamnionitis was verified by histological findings of the placenta

c $\mathrm{PDA}=$ haemodynamic relevant patent ductus arteriosus

$\mathrm{d}$ PIP = maximum peak inspiratory pressure

e Cord blood $\mathrm{pH}=$ arterial chord blood $\mathrm{pH}: 1$ defined as $<7.15$ and 0 defined as $>7.15$

$\mathrm{f}$ comparable results were obtained for APGAR-scores at 1 and 5 minutes

$\mathrm{g}$ Contact to other children $=$ having siblings or going to a nursery care institution regularly

factors reflecting perinatal adaptation, such as APGARscore, CRIB-score and cord blood $\mathrm{pH}$.

\section{RESPIRATORY MORBIDITY}

Data on prevalence of cough and wheeze in preterm infants from the post-surfactant era are rare: Greenough et al. reported that $51 \%$ of preterm infants born at less than 29 weeks of gestation suffered from cough and $42 \%$ from wheeze during the first year of life [8]. Vrijland et al. described cough to occur in $97 \%$ and wheeze in $39 \%$ of preterm infants [21]. In an important study from the presurfactant era 53 to $65 \%$ of the infants had cough or wheeze during the first 6 months of life [22]. Our results are well in line with these reports. Interestingly, despite differences in data assessment, age range, observational periods and countries, the high prevalence of wheeze and even higher prevalence of cough seems to be a consistent finding among studies.

Re-hospitalisation rates of preterm infants in the first 1-2 years are reported in the literature to occur in $14 \%$ [23] to $38 \%$ [24] of preterm infants with and without BPD, well comparable to our results and known to be dependent on gestational age and differences in patient management [24].
To our knowledge there are no data available comparing respiratory morbidity between term- and preterm-born infants from the same area and time period. We have recently reported on respiratory morbidity and risk factors in a large unselected prospective cohort of term-born infants with weekly assessment of respiratory symptoms [6]. Recruitment area and time period were the same as in the present study. In these healthy term-born infants, the overall prevalence of cough was comparable (93\%, compared to $79 \%$ in preterm children), whereas wheeze occurred less often in term children (21\%) than in preterm children (44\%). This is quite remarkable, considering that the method of data collection used for term infants (weekly phone calls) is expected to be more sensitive than the method used for preterm children (retrospective questionnaire) [12]. Hospitalisation due to respiratory reasons in the first year of life was reported for $25 \%$ of preterm, but only for $1.5 \%$ of term-born infants [6].

\section{RISK FACTORS}

Common symptoms were mainly associated with environmental exposures, in accordance with findings by Greenough et al. who reported that male sex, duration of oxygen therapy, older siblings and socioe- 
conomic status were associated with respiratory symptoms in the first year of life in preterms born before 29 weeks of gestation [8]. Our results show that this holds also for preterms born after 29 weeks of gestation. In contrast, severe respiratory symptoms were stronger associated with parameters reflecting perinatal adaptation. APGAR- and CRIB score have been described to predict outcome of preterm infants [19] and it has been shown that higher PIP is associated with decreased survival rates [17].

Surprisingly, BPD was not correlated with severe respiratory morbidity in the present study, although a trend was found for severe outcomes. The association with BPD severity and long-term respiratory morbidity has remained controversial and comparison of these studies is difficult due to varying BPD definitions [25-28]. Ehrenkranz et al. found a reasonable association between severe outcomes and the "new BPD" classification [3]. One possible explanation might be differing neonatal treatment strategies between Europe and the USA. Premature infants in our cohort had shorter mechanical ventilation and early nasal CPAP therapy. In contrast, different guidelines lead to intubation and longer duration of mechanical ventilation in the USA [29].

Gestational age was not associated with respiratory symptoms. These results are again different than American results [30] but in line with the London cohort [8]. This might reflect the different picture of "new" BPD, which is indeed known not to be exclusively dependent on gestational age [8].

While risk factors for common respiratory symptoms were similar for premature and term neonates, severe outcomes in preterms were related to perinatal factors. Furthermore, other factors found to be associated with respiratory morbidity in the term-born cohort, such as sex, birthweight and maternal atopy were not associated with respiratory symptoms in preterms. We assume that the influence of these factors is masked by stronger effects induced by prematurity and the related developmental changes of the immune system as well as of lung and airway growth. It is e.g. known that boys have smaller airways at birth and thus more respiratory problems than girls [31], but this gender difference is less prominent in premature infants [32] and likely to be overruled by other factors.

\section{Parental Perception}

One third of the parents may overestimate the common respiratory morbidity of their preterm born children when comparing to term infants and consult their Paediatrician early. Thus parental counselling at discharge related to common in respect to severe respiratory morbidity may help to improve the burden of the health care system.

\section{Methodological Issues}

There are limitations of our study. It is a retrospective survey, with questionnaires sent to the parents at different ages of their children. This may lead to recall bias, however similar results after stratification by age at reply of questionnaires makes this unlikely. Furthermore it has been shown that questions on respiratory symptoms have a good repeatability, supporting validity of our findings $[13,33]$. Another possible bias is the fact that we only included children who underwent infant lung function which makes generalisation of the results more difficult. Due to rather low numbers we were not able to perform multivariable analyses. The relatively small sample size might also explain why parameters like gestational age or chorioamnionitis were only weakly associated with respiratory morbidity [3]. In addition, causal pathways are difficult to model in the analysis, because many of the examined parameters may function either as independent risk factor or alternatively be a consequence of another risk factor, therefore lying in the causal pathway. For instance high peak inspiratory pressure can injure the premature lung by itself, on the other hand, ventilation with high pressures could have been made necessary because of an injured premature lung. All these factors are important to bear in mind when interpreting the results. To validate our findings and clearly disentangle all these interactions, larger studies should be conducted in future.

\section{RELEVANCE}

From a clinical point of view, the identification of risk factors may help to promote further individualized treatment and prevent long-term sequelae in preterm infants. The risk factors for respiratory diseases during infancy should be considered as an important part of planning discharge and follow-up of high-risk premature infants. For instance, in line with other, mainly European studies, we can support the hypothesis that gentle breathing support results in less frequent BPD rates as well as in less respiratory symptoms even in very young preterm infants [34].

From a public health point of view, prenatal birth is the commencement of a relevant burden of disease for patients, parents [35] and the health care system. This includes doctor visits apart from routine checkups, re-hospitalisations as well as psychological and financial burden of long-term medication. This burden is strongly dependent on the correct identification of those infants subject to appropriate treatment. In Switzerland, decisions on medication and reimbursement of health care costs are strongly influenced by the current BPD definition. For instance, vaccination against respiratory syncytial virus is only recommended and paid in preterm infants with severe BPD, it can be considered for patients with moderate BPD and is not recommended for patients with mild BPD. However, our data and other studies show that prematurity rather than BPD definitions and other risk factors define respiratory morbidity in these preterm infants in the first year of life. Due to these aspects current health care policies need urgent reconsideration.

\section{CONCLUSION}

The results of this study indicate that premature infants are subject to a high incidence of respiratory morbidity even after hospital discharge imposing a rel- 
evant burden of disease for patients, parents and the health care system. By describing respiratory morbidity in the first year of life in these preterm infants and assessing associated risk factors, our study will contribute to the identification of patients with the highest risk for later respiratory morbidity. Growing knowledge on the pathophysiology as well as changes in perinatal management will however lead to continuous changes of this picture. As more patients with the "new" form of BPD will reach older age, larger studies with longer follow-up times and measurement of subtle lung function indices will be needed to assess the long-term outcome of these children.

Acknowledgements

O Ahrens, MD, NEODAT administrator at the division of neonatology at the University Children's hospital of Bern.

\section{REFERENCES}

1. Northway WH, Jr., Rosan RC, Porter DY. Pulmonary disease following respirator therapy of hyaline-membrane disease. Bronchopulmonary dysplasia. N Engl J Med 1967; 276(7):357-68.

2. Jobe AH, Bancalari E. Bronchopulmonary dysplasia. Am J Respir Crit Care Med 2001;163(7):1723-9.

3. Ehrenkranz RA, Walsh MC, Vohr BR, Jobe AH, Wright LL, Fanaroff AA, et al. Validation of the National Institutes of Health consensus definition of bronchopulmonary dysplasia. Pediatrics 2005;116(6):1353-60.

4. Belanger K, Beckett $\mathrm{W}$, Triche E, Bracken MB, Holford $\mathrm{T}$, Ren $\mathrm{P}$, et al. Symptoms of wheeze and persistent cough in the first year of life: associations with indoor allergens, air contaminants, and maternal history of asthma. Am J Epidemiol 2003;158(3):195-202.

5. Kusel MM, de Klerk NH, Holt PG, Kebadze T, Johnston SL, Sly PD. Role of respiratory viruses in acute upper and lower respiratory tract illness in the first year of life: a birth cohort study. Pediatr Infect Dis J 2006;25(8):680-6.

6. Latzin P, Frey U, Roiha HL, Baldwin DN, Regamey N, Strippoli MP, et al. Prospectively assessed incidence, severity, and determinants of respiratory symptoms in the first year of life. Pediatr Pulmonol 2007;42(1):41-50.

7. Gross SJ, Iannuzzi DM, Kveselis DA, Anbar RD. Effect of preterm birth on pulmonary function at school age: a prospective controlled study. J Pediatr 1998;133(2):18892.

8. Greenough A, Limb E, Marston L, Marlow N, Calvert S, Peacock J. Risk factors for respiratory morbidity in infancy after very premature birth. Arch Dis Child Fetal Neonatal Ed 2005;90(4):F320-3.

9. Bush A. COPD: a pediatric disease. Copd 2008;5(1):5367.

10. Greenough A. Long-term pulmonary outcome in the preterm infant. Neonatology 2008;93(4):324-7.

11. Aukland SM, Rosendahl K, Owens CM, Fosse KR, Eide GE, Halvorsen T. Neonatal bronchopulmonary dysplasia predicts abnormal pulmonary HRCT scans in long-term survivors of extreme preterm birth. Thorax 2009;64(5): 405-10.

12. Asher MI, Keil U, Anderson HR, Beasley R, Crane J, Martinez F, et al. International Study of Asthma and Allergies in Childhood (ISAAC): rationale and methods. Eur Respir J 1995;8(3):483-91.

13. Strippoli MP, Silverman M, Michel G, Kuehni CE. A parent-completed respiratory questionnaire for 1-year-old children: repeatability. Arch Dis Child 2007;92(10):861-5.

14. McLeod A, Ross P, Mitchell S, Tay D, Hunter L, Hall A, et al. Respiratory health in a total very low birthweight cohort and their classroom controls. Arch Dis Child 1996;74(3):188-94.

15. Watterberg KL, Demers LM, Scott SM, Murphy S. Chorioamnionitis and early lung inflammation in infants in whom bronchopulmonary dysplasia develops. Pediatrics 1996;97(2):210-5.

16. Rojas MA, Gonzalez A, Bancalari E, Claure N, Poole C, Silva-Neto G. Changing trends in the epidemiology and pathogenesis of neonatal chronic lung disease. J Pediatr 1995;126(4):605-10.

17. Gray PH, Grice JF, Lee MS, Ritchie BH, Williams G. Prediction of outcome of preterm infants with severe bronchopulmonary dysplasia. J Paediatr Child Health 1993; 29(2):107-12.

18. Cunha GS, Mezzacappa-Filho F, Ribeiro JD. Risk factors for bronchopulmonary dysplasia in very low birth weight newborns treated with mechanical ventilation in the first week of life. J Trop Pediatr 2005;51(6):334-40.

19. Forsblad K, Kallen K, Marsal K, Hellstrom-Westas L. Apgar score predicts short-term outcome in infants born at 25 gestational weeks. Acta Paediatr 2007;96(2):16671.

20. DiFranza JR, Aligne CA, Weitzman M. Prenatal and postnatal environmental tobacco smoke exposure and children's health. Pediatrics 2004;113(4 Suppl):1007-15.

21. Vrijlandt EJLE, Boezen HM, Gerritsen J, Stremmelaar FF, Duiverman EJ. Respiratory health in prematurely born preschool children with and without bronchopulmonary dysplasia. Journal of Pediatrics 2007;150(3):256261.

22. Greenough A, Maconochie I, Yuksel B. Recurrent respiratory symptoms in the first year of life following preterm delivery. J Perinat Med 1990;18(6):489-94.

23. Resch B, Pasnocht A, Gusenleitner W, Muller W. Rehospitalisations for respiratory disease and respiratory syncytial virus infection in preterm infants of 29-36 weeks gestational age. J Infect 2005;50(5):397-403.

24. Blondel B, Truffert P, Lamarche-Vadel A, Dehan M, Larroque $\mathrm{B}$. [Use of medical services by very preterm children during the first year of life in the Epipage cohort]. Arch Pediatr 2003;10(11):960-8.

25. Greenough A. Measuring respiratory outcome. Semin Neonatol 2000;5(2):119-26.

26. Greenough A, Cox S, Alexander J, Lenney W, Turnbull F, Burgess S, et al. Health care utilisation of infants with chronic lung disease, related to hospitalisation for RSV infection. Arch Dis Child 2001;85(6):463-8.

27. Gregoire MC, Lefebvre F, Glorieux J. Health and developmental outcomes at 18 months in very preterm infants with bronchopulmonary dysplasia. Pediatrics 1998;101(5): 856-60.

28. Furman L, Baley J, Borawski-Clark E, Aucott S, Hack M. Hospitalization as a measure of morbidity among very low birth weight infants with chronic lung disease. J Pediatr 1996;128(4):447-52.

29. Vanpee M, Walfridsson-Schultz U, Katz-Salamon M, Zupancic JA, Pursley D, Jonsson B. Resuscitation and ventilation strategies for extremely preterm infants: a comparison study between two neonatal centers in Boston and Stockholm. Acta Paediatr 2007;96(1):10-6; discussion 8-9.

30. Kumar R, Yu Y, Story RE, Pongracic JA, Gupta R, Pearson C, et al. Prematurity, chorioamnionitis, and the development of recurrent wheezing: a prospective birth cohort study. J Allergy Clin Immunol 2008;121(4):878-84 e6.

31. Hibbert M, Lannigan A, Raven J, Landau L, Phelan P. Gender differences in lung growth. Pediatr Pulmonol 1995;19(2):129-34.

32. Stocks J, Henschen M, Hoo AF, Costeloe K, Dezateux C. Influence of ethnicity and gender on airway function in preterm infants. Am J Respir Crit Care Med 1997;156(6): 1855-62. 
33. Kuehni CE, Brooke AM, Silverman M. Prevalence of wheeze during childhood: retrospective and prospective assessment. Eur Respir J 2000;16(1):81-5.

34. Ramanathan R. Optimal ventilatory strategies and surfactant to protect the preterm lungs. Neonatology 2008; 93(4):302-8.

35. McLean A, Townsend A, Clark J, Sawyer MG, Baghurst P, Haslam R, et al. Quality of life of mothers and families caring for preterm infants requiring home oxygen therapy: a brief report. J Paediatr Child Health 2000;36(5): 440-4.
Received: May 2, 2010 / Accepted: July 20, 2010

Address fir correspondence:

Isabelle Pramana

Devision of paediatric pneumology,

Children's Hospital of the University of Bern

3010 Bern

Switzerland

Phone: +41-31-632 2111

Fax: +41-31-632 4807

E-mail: isabellepramana@gmail.com 